\title{
First order hyperbolic formalism for numerical relativity
}

\author{
C. Bona \\ Departament de Física, Universitat de les Illes Balears, E-07071 Palma de Mallorca, Spain \\ J. Massó \\ Max-Planck-Institut für Gravitationsphysik, Schlaatzweg 1, D-14473 Potsdam, Germany \\ and National Center for Supercomputing Applications, Beckman Institute, 405 North Mathews Avenue, Urbana, Illinois 61801 \\ E. Seidel \\ Max-Planck-Institut für Gravitationsphysik, Schlaatzweg 1, D-14473 Potsdam, Germany; \\ National Center for Supercomputing Applications, Beckman Institute, 405 North Mathews Avenue, Urbana, Illinois 61801; \\ and Departments of Physics and Astronomy, University of Illinois, Urbana, Illinois 61801 \\ J. Stela \\ Departament de Física, Universitat de les Illes Balears, E-07071 Palma de Mallorca, Spain
}

(Received 24 March 1997)

\begin{abstract}
The causal structure of Einstein's evolution equations is considered. We show that in general they can be written as a first-order system of balance laws for any choice of slicing or shift. We also show how certain terms in the evolution equations, which can lead to numerical inaccuracies, can be eliminated by using the Hamiltonian constraint. Furthermore, we show that the entire system is hyperbolic when the time coordinate is chosen in an invariant algebraic way, and for any fixed choice of the shift. This is achieved by using the momentum constraints in such a way that no additional space or time derivatives of the equations need to be computed. The slicings that allow hyperbolicity in this formulation belong to a large class, including harmonic, maximal, and many others that have been commonly used in numerical relativity. We provide details of some of the advanced numerical methods that this formulation of the equations allows, and we also discuss certain advantages that a hyperbolic formulation provides when treating boundary conditions. [S0556-2821(97)05616-6]
\end{abstract}

PACS number(s): 04.25.Dm

\section{INTRODUCTION AND OVERVIEW}

In a previous Letter [1], we proposed a new formalism for numerical relativity based on a formulation of Einstein field equations as a hyperbolic system of balance laws. This was an extension of previous results which were derived originally in a particular gauge (harmonic slicing) [2], but in [1] we showed how to enlarge this to a broad family of slicing conditions, including the most commonly used choices in numerical relativity. The application of this formalism to practical problems requires a more detailed description and discussion, which is the aim of the present work. In this first follow-up paper we describe the formalism in much more detail than before, and describe broadly the kinds of numerical methods that are applicable to such a system of equations. In future papers in this series we will provide detailed numerical examples in one dimension (1D) and 3D, with comparisons to other formalisms and standard numerical methods.

There are numerous motivations for this new formulation of the equations.

(i) Numerical methods. First, standard numerical methods for evolution systems, such as flux conservative balance laws, have been developed specifically to treat only certain systems of equations, and only for these systems are their numerical properties well understood. The standard Arnowitt-Deser-Misner (ADM) formulation of the equations
[3-5] is much more complicated, and therefore one has to be very careful when attempting to apply ad hoc variations on these methods to Einstein's theory; the numerical properties of these systems are not well understood. This is a major reason why numerical relativity has proved much more difficult than, say, computational fluid dynamics (CFD). For example, without novel approaches, such as apparent horizon boundary conditions [6,7], black hole spacetimes, could not previously be evolved beyond about $t=150 M[8,9]$ without codes crashing due to the inadequacy of the numerical methods being used. Worse yet, in interesting cases where black holes (i.e., horizons) do not seem to form, yet where singularities may be developing, similar problems cause codes to become very inaccurate and crash even much earlier [10], preventing a full exploration of the spacetime. If special numerical methods could be developed specifically for the standard formulation of the Einstein equations, as they have been for decades in CFD, presumably these problems could be treated properly. However, this would be a formidable undertaking. On the other hand, we have managed to write the equations in a form which can take advantage of this vast knowledge of numerical methods applied to systems of conservations laws, and their numerical properties, developed for CFD. With this new formulation one can now apply many standard methods (e.g., the genuine MacCormack [12] method) for any choice of lapse and shift. This already offers new possibilities for evolution schemes. But for a wide fam- 
ily of lapse conditions, the system is furthermore hyperbolic, meaning here that one can find a complete set of eigenfields with real eigenvalues for the system of equations. This provides a framework for developing a much deeper knowledge of the system of equations, its characteristic fields and their speeds, and also brings a variety of more advanced numerical methods [e.g., so-called total variation diminishing (TVD) schemes [13]] that exploit this knowledge at the finite difference level. In CFD this knowledge is crucial in treating the kinds of large gradients and "shocklike" features that can also develop in strongly gravitating systems due to gauge or physical effects in the Einstein system. We will give examples of these possibilities below.

(ii) Boundary conditions. Second, in the case of slicings that allow the system to be hyperbolic, the decomposition of the system into its eigenfields can be crucial in developing appropriate boundary conditions. On a finite domain, boundaries have always been a serious problem in numerical relativity. But in a hyperbolic system one has detailed knowledge of which quantities are propagating in which directions, and also their speeds. This allows a natural identification of radiative variables. This information is crucial in formulating conditions at boundaries that allow outgoing quantities to actually escape from the system ("outgoing radiation conditions"), while providing ways to avoid generating unphysical and unwanted signals that propagate inward from the boundaries ("no incoming radiation conditions"). This is useful not only at the outer boundary, but also it may be especially important in the case of black holes where boundary conditions are imposed on the horizon ["apparent horizon boundary conditions," (AHBC)]. In AHBC's, which exploits the causal properties of the spacetime to chop out singular regions inside the black hole, a detailed knowledge of the causal structure of the entire system of equations is very important, and can be provided through a hyperbolic treatment.

(iii) Gauge modes. Another important aspect of this way of writing the system is the identification of gauge modes. For slicings that ensure hyperbolicity, not only are physical degrees of freedom identified, which must propagate at the speed of light, but also special gauge modes, which are related to the choice of slicing, are naturally singled out. This separation of physical from gauge effects may play an important role in devising appropriate gauges or in interpreting numerical results.

(iv) Theoretical analysis. Because hyperbolic systems of conservation laws have been studied for many years, much is known about their theoretical properties, the existence of solutions, the treatment of boundaries, the conditions under which shocks can develop, etc. [14-18]. With the entire set of Einstein equations now cast in this form, for a variety of practical gauge conditions, they can be studied with this body of knowledge in mind.

The starting point for this new formulation is the standard $3+1$ decomposition of spacetime [3-5], which clearly separates the dynamical degrees of freedom from the gauge ones: the lapse function $\alpha$ and the shift components $\beta^{i}$. In our previous Letter [1] we considered in detail only the zero shift case just for simplicity. We complete here the presentation by considering the arbitrary shift case. The term "arbitrary" here means that one can prescribe the shift as a given space- time function. It does not mean that one can naively prescribe it by a local relationship with dynamical quantities (as one does with the lapse when choosing, for instance, harmonic slicing), because this would turn the shift into a dynamical quantity and then the hyperbolicity of the complete system would hold only for some specific choices. We will instead keep the shift as a purely kinematical degree of freedom which can be adapted to every specific problem. This is a subtle, but important, point that will be discussed further below.

In order to cast the evolution system into first-order hyperbolic form, we introduce three extra dynamical quantities $V_{i}$ with evolution equations provided by the momentum constraint. This approach is different from the classical one, where harmonic coordinates are enough to get hyperbolicity [19]. This is the price to pay for having an arbitrary shift, because one can no longer use the three shift components to eliminate unwanted terms in Einstein equations. The gaugeindependent alternative of using the three momentum constraints is more adapted to numerical applications.

This use of the momentum constraints is a shared feature in many new hyperbolic formalisms [20,21]. Some of these [20] are variations on the idea from Choquet and Ruggeri [22] of taking an extra time derivative to get a third-order evolution system which can then be written into first-order form. Others prefer to take an extra space derivative of the Einstein field equations [23] to take advantage of the Bianchi identities. In any case, the extra derivatives multiply the number of independent quantities to be evolved. Our approach, instead, uses only three extra quantities and contains no extra derivative of any kind. In many senses, it is similar to the one recently developed by Fritelli and Reula [21].

Another shared feature in all the new hyperbolic formalisms is that all of them allow harmonic slicing of the spacetime (harmonic time coordinate). This implies a local relationship between the lapse function and the space volume element, so that the lapse becomes a dynamic degree of freedom. In our formalism, we generalize the harmonic condition to a much wider set of dynamical slicings, including the ones which have been actually used in successful numerical applications [24,25]. To be more specific, let us remember that in the zero shift case, harmonic slicing amounts to a linear relationship between lapse and space volume element, whereas our general case amounts to the lapse being any monotonically increasing function of the space volume element. An interesting point is that this condition not only ensures hyperbolicity, but also singularity avoidance. We extend below to our general case a previous proof for harmonic slicing [26].

In a recent work [27], the question of whether a dynamical gauge condition can introduce the so-called "coordinate shocks" has been raised. Although our formalism [1] has been used for simplicity to illustrate the point, this problem is inherent to harmonic slicing itself, and therefore to all formulations of the equations, whether they follow the ADM approach, the new hyperbolic formalisms [20,21,23], or any other formulation. The same problem has been detected even in numerical codes based in nonhyperbolic systems but using dynamical gauges [28]. More work is needed to understand the implications of that effect, but in any case a way around this problem is to use maximal slicing [29]. In that way one 
gets a coupled elliptic-hyperbolic system in which the gauge degree of freedom is elliptic so no coordinate shocks can appear due to the slicing. This could explain why codes based on maximal slicing are usually more robust.

A final important point in our introduction is the choice of an evolution system. In our previous Letter [1] we considered only the standard choice, arising from the space components of the Ricci tensor $R_{a b}$. As we detail in Sec. II B below, there is actually a one-parameter family of physically equivalent evolution systems which are all hyperbolic for the same gauge choices with the same characteristic speeds: we provide the eigenvectors for all of them. Far from being a mathematical curiosity, this choice freedom allows one to select the system in that family which is free from Newtonian contributions. By this we mean that the evolution of the gravitational field is a purely relativistic effect: in Newtonian gravity there is no evolution and the gravitational field can be computed at every instant by integrating an elliptic equation (the Poisson equation, which can be understood as the Newtonian limit of the Hamiltonian constraint). In all but one of the evolution systems in that family the general relativistic dynamical terms are mixed with pure Newtonian contributions. The only evolution system in which this does not happen turns out to be different from the standard one: it arises from the space components of the Einstein tensor $G_{a b}$ and then we will call it the "Einstein system." This is crucial for numerical applications either to weak field problems or at the outer boundary of a finite difference grid.

\section{THE FORMALISM}

\section{A. Space plus time decomposition}

In order to clarify the differences between the new evolution system we propose and its predecessors, we first review the standard evolution system. The Einstein field equations consist of a nonlinear system of ten second order partial differential equations when written in terms of the spacetime metric components $g_{\mu \nu}$. In order to study the causal structure of this system, we will use a time coordinate $t$ to label the evolution. This amounts to introducing a "lapse" function $\alpha$ relating $d t$ with the proper time interval between the $t=$ const hypersurfaces.

The following study depends on the actual choice of this time coordinate, so that we will consider changes of the space coordinates only. In this sense, it is clear that the lapse function $\alpha$ is a scalar quantity and the 3D "induced metric" $\gamma_{i j}$ on every constant $t$ hypersurface is a tensor quantity. It is then more convenient to write down the line element in the following way $(3+1$ decomposition [3-5]):

$$
d s^{2}=-\alpha^{2} d t^{2}+\gamma_{i j}\left(d x^{i}+\beta^{i} d t\right)\left(d x^{j}+\beta^{j} d t\right),
$$

where the shift $\beta^{i}$ is related to the choice of space coordinates on every $t=$ const hypersurface.

Another important tensor quantity is the extrinsic curvature $K_{i j}$ (second fundamental form) of the hypersurfaces, which can be expressed just as the proper time derivative of the induced metric, taken along the normal lines:

$$
\left(\partial_{t}-\mathcal{L}_{\beta}\right) \gamma_{i j}=-2 \alpha K_{i j}
$$

Einstein's equations can then be expressed in terms of the set of variables

$$
\left(\alpha, \beta^{i}, \gamma_{i j}, K_{i j}\right)
$$

and it can be seen that no time derivative of the lapse nor the shift appears into the resulting system. So far this is just the standard 3+1 approach [3-5].

We can either consider these kinematical quantities $\alpha, \beta^{i}$ as being arbitrarily prescribed or we can provide supplementary equations for them. In this work we shall take a combined approach, by supposing that the shift components $\beta^{i}$ are known spacetime functions (we took them to be zero in our previous Letter [1] just for simplicity), whereas we choose to evolve the lapse $\alpha$ according to

$$
\left(\partial_{t}-\beta^{k} \partial_{k}\right) \ln \alpha=-\alpha Q,
$$

where the function $Q$ will be given later. This form will turn out to encompass many common choices of lapse.

\section{B. Evolution systems}

The evolution of $K_{i j}$ is given by a set of six evolution equations obtained from Einstein equations. For instance, the space components of the four-dimensional Ricci tensor ${ }^{(4)} R_{i j}$ can be written [4]

$$
\left(\partial_{t}-\mathcal{L}_{\beta}\right) K_{i j}=-\alpha_{i ; j}+\alpha\left[{ }^{(3)} R_{i j}-2 K_{i j}^{2}+\operatorname{tr} K K_{i j}-{ }^{(4)} R_{i j}\right],
$$

where index contractions and covariant derivatives are with respect to the induced metric $\gamma_{i j}$, and the three-dimensional Ricci tensor constructed from the induced metric is denoted by ${ }^{(3)} R_{i j}$. This set of equations, together with Eq. (2), is taken to be the standard evolution system. We shall call it in what follows the "Ricci evolution system" for the set of variables (3).

The remaining four Einstein equations are constraints, which can be easily identified: the "energy," or Hamiltonian constraint

$$
2 \alpha^{2} G^{00}={ }^{(3)} R+(\operatorname{tr} K)^{2}-\operatorname{tr}\left(K^{2}\right),
$$

where ${ }^{(3)} R$ is the trace of the three-dimensional Ricci tensor, and the "momentum constraint"

$$
\alpha G_{i}^{0}=K_{i ; k}^{k}-\partial_{i}(\operatorname{tr} K) .
$$

These constraint equations are first integrals of the evolution system. They are then redundant provided that they are imposed on the initial data (otherwise one would get unphysical solutions).

It is not often appreciated that, although it is the standard evolution system, the Ricci system (5) is not convenient for many numerical applications. One way of seeing this is to look at the space components of the Ricci tensor for a perfect fluid:

$$
{ }^{(4)} R_{i j}=8 \pi\left[(\mu+p) u_{i} u_{j}+1 / 2(\mu-p) \gamma_{i j}\right] .
$$

where $\mu$ is the total energy density of the fluid, $p$ is the pressure, and $u_{i}$ is its fluid three-velocity. Notice that the second term contains a contribution from the energy of the 
fluid which does not vanish in the Newtonian limit, where the three-velocities are taken to be small compared to one and the pressure small compared to the energy density.

This means that the system (5) has a Newtonian contribution from the energy density which, allowing for the energy constraint (6), is to be compensated with other Newtonian contributions in the geometry terms. This situation can be very inconvenient either in "post-Newtonian" (moderately relativistic) scenarios, where the small relativistic evolution effects can easily be masked by the truncation errors of the larger Newtonian terms, or even in strong field scenarios where it can be difficult to preserve the constraints at the boundaries.

To remedy this problem, let us note that an evolution equation plus a constraint leads to another evolution equation. Using the relation

$$
{ }^{(4)} R_{i j}=G_{i j}-1 / 2\left(-\alpha^{2} G^{00}+\operatorname{tr} G\right) \gamma_{i j},
$$

where we have noted $\operatorname{tr} G=\gamma^{i j} G_{i j}$, we will combine the energy constraint (6) with Eq. (5) to cancel out the $G^{00}$ term, obtaining a different evolution system,

$$
\begin{aligned}
\left(\partial_{t}-\mathcal{L}_{\beta}\right) K_{i j}= & -\alpha_{i ; j}+\alpha\left[{ }^{(3)} R_{i j}-2 K_{i j}^{2}+\operatorname{tr} K K_{i j}-G_{i j}\right] \\
& -\alpha / 4 \gamma_{i j}\left[{ }^{(3)} R-\operatorname{tr}\left(K^{2}\right)+(\operatorname{tr} K)^{2}-2 \operatorname{tr} G\right]
\end{aligned}
$$

which is equivalent to the one arising from the space components $G_{i j}$ of the Einstein tensor.

We shall call it in what follows the "Einstein evolution system." The matter terms in the perfect fluid case can be computed now from

$$
G_{i j}=8 \pi\left[(\mu+p) u_{i} u_{j}+p \gamma_{i j}\right]
$$

so that they vanish in the Newtonian limit. This "Einstein system" has been found to be useful in tests of hydrodynamic evolution [11], but it is important not only in the matter case. The use of the Einstein system (10) turns out to be very important to obtain the long term evolution for a vacuum 1D black hole that we presented in our previous Letter, and will be discussed in detail in a future paper in this series.

The two systems (5) and (10) are not equivalent: they can, in principle, have different solutions. However, the physical solutions (the ones verifying the constraints) are common to both systems. Physics is not affected, of course, but the mathematical structure can be modified by the choice of the evolution system among the infinitely many combinations of the Ricci system with the energy and/or momentum constraints. As we have suggested that the use of the energy constraint is important for accuracy, we will see below that the use of the momentum constraint is crucial to ensure hyperbolicity [22,2]. This provides a posteriori a good criterion for choosing a particular evolution system among the many possibilities.

\section{A first-order evolution system}

The evolution systems (5),(10) are first order in time, but second order in space. To obtain systems which are also of first order in space, we will follow the standard procedure by introducing auxiliary variables which correspond to the space derivatives:

$$
A_{k}=\partial_{k} \ln \alpha, \quad B_{k}^{i}=1 / 2 \partial_{k} \beta^{i}, \quad D_{k i j}=1 / 2 \partial_{k} \gamma_{i j}
$$

Note that the shift components are given at every instant so that the space derivatives $B_{i}^{j}$ are known. The evolution equations for the remaining quantities can be obtained by taking the time derivative of Eq. (12) and interchanging the order of space and time derivatives:

$$
\begin{gathered}
\partial_{t} A_{k}+\partial_{k}\left[-\beta^{r} A_{r}+\alpha Q\right]=0, \\
\partial_{t} D_{k i j}+\partial_{k}\left[-\beta^{r} D_{r i j}+\alpha\left(K_{i j}-s_{i j}\right)\right]=0,
\end{gathered}
$$

where we have used the shorthand

$$
s_{i j}=\left(B_{i j}+B_{j i}\right) / \alpha,
$$

and for notational convenience, we have also written $B_{i j}=\gamma_{i k} B_{j}^{k}$, even though $B_{i j}$ is not a tensor quantity.

Note that we have used here the ordering freedom of space derivatives in a different way than we did in our previous Letter [1], where Eq. (14) was written as

$$
\begin{gathered}
\partial_{t} D_{k i j}+\partial_{r}\left[-\beta^{r} D_{k i j}+\alpha \delta_{k}^{r}\left(K_{i j}-s_{i j}\right)\right] \\
=\left(2 B_{k}^{r}-\alpha \operatorname{tr} s \delta_{k}^{r}\right) D_{r i j} .
\end{gathered}
$$

The present choice (14) is more suitable for numerical applications when the shift does not vanish, as it does not introduce extra sources. The same criterion leads us to write down Eqs. (2),(4) as

$$
\begin{gathered}
\partial_{t} \gamma_{i j}=-2 \alpha\left(K_{i j}-s_{i j}\right)+2 \beta^{r} D_{r i j}, \\
\partial_{t} \ln \alpha=-\alpha Q+\beta^{r} A_{r} .
\end{gathered}
$$

So far, Eqs. (13)-(17) have been written in a first order balance law form

$$
\partial_{t} \mathbf{u}+\partial_{k} F_{-}^{k} \mathbf{u}=S_{-} \mathbf{u}
$$

where the vector $\mathbf{u}$ displays the set of variables and both "fluxes" $F^{k}$ and "sources" $S$ are vector valued functions. Our goal will be write the entire system of evolution equations in this form. We introduce the additional quantities

$$
V_{i}=D_{i r}^{r}-D_{r i}^{r}
$$

where again even though the $D_{i j k}$ are not components of a tensor, we raise indices in the usual way with the threemetric $\gamma_{i j}$. Then after extensive manipulation the evolution equations for the extrinsic curvature components (5) can also be put in the first-order balance law form given by Eq. (18).

This almost completes the system, for which the nonzero fluxes are

$$
\begin{gathered}
F_{-}^{k} A_{k}=-\beta^{r} A_{r}+\alpha Q, \\
F_{-}^{k} D_{k i j}=-\beta^{r} D_{r i j}+\alpha\left(K_{i j}-s_{i j}\right),
\end{gathered}
$$




$$
\begin{aligned}
F_{-}^{k} K_{i j}= & -\beta^{k} K_{i j}+\alpha\left[D_{i j}^{k}-n / 2 V^{k} \gamma_{i j}+\frac{1}{2} \delta_{i}^{k}\left(A_{j}+2 V_{j}-D_{j r}^{r}\right)\right. \\
& \left.+\frac{1}{2} \delta_{j}^{k}\left(A_{i}+2 V_{i}-D_{i r}^{r}\right)\right],
\end{aligned}
$$

where the free parameter $n$ allows one to select a specific evolution system (it is zero for the Ricci system and one for the Einstein system). The nonzero source terms are those appearing in Eq. (17) and

$$
\begin{aligned}
S_{-} K_{i j}= & 2\left(K_{i r} B_{j}^{r}+K_{j r} B_{i}^{r}-K_{i j} B_{r}^{r}\right)+\alpha\left\{-{ }^{(4)} R_{i j}-2 K_{i}^{k} K_{k j}\right. \\
& +\operatorname{tr} K K_{i j}-\Gamma_{r i}^{k} \Gamma_{k j}^{r}+2 D_{i k}^{r} D_{r j}^{k}+2 D_{j k}^{r} D_{r i}^{k}+\Gamma_{k r}^{k} \Gamma_{i j}^{r} \\
& -\left(2 D_{k r}^{k}-A_{r}\right)\left(D_{i j}^{r}+D_{j i}^{r}\right)+A_{i}\left(V_{j}-1 / 2 D_{j k}^{k}\right)+A_{j}\left(V_{i}\right. \\
& \left.-1 / 2 D_{i k}^{k}\right)-n V^{k} D_{k i j}+n / 4 \gamma_{i j}\left[-D_{k}^{r s} \Gamma_{r s}^{k}+D_{k r}^{r} D_{s}^{k s}\right. \\
& \left.\left.-2 V^{k} A_{k}+\operatorname{tr}\left(K^{2}\right)-(\operatorname{tr} K)^{2}+2 \alpha^{2} G^{00}\right]\right\} .
\end{aligned}
$$

So far we have achieved a balance law formulation which will be valid for any gauge choice (lapse and shift). Already this is something useful, as many numerical methods, such as the genuine MacCormack scheme [12], have been devised explicitly for such a system. In particular we note that without this balance law formulation, one cannot apply a method like MacCormack, which was designed to treat not only the time evolution, but also the fluxes and sources, in a specific way. Previous applications of a "MacCormack-like" method, as in Ref. [8], used only the time evolution part of this scheme, leaving the most important spatial part of the system to be treated without regard for the particular structure of the equations.

However, as important as the balance law formulation is, additional benefit could be gained if the system would be actually hyperbolic. This means that the entire system of balance law equations (18) can be diagonalized, with a complete set of eigenvectors with real eigenvalues. This is not yet the case, mainly because of the combinations (19) arising in the flux terms (20). They could be eliminated by a suitable shift choice (imposing harmonic space coordinates, for instance), but we prefer to deal with an arbitrary shift and we will proceed in a different way.

The three combinations defined by Eq. (19) are very interesting quantities. One can actually compute their time derivative from Eq. (14) and make use of the momentum constraint, (7) to obtain for these three combinations evolution equations of the balance law form (18) with

$$
\begin{gathered}
F_{-}^{k} V_{i}=-\beta^{k} V_{i}+B_{i}^{k}-B_{i}^{k}, \\
S_{-} V_{i}=\alpha\left[\alpha G_{i}^{0}+A_{r}\left(K_{i}^{r}-\operatorname{tr} K \delta_{i}^{r}\right)+K_{s}^{r}\left(D_{i r}^{s}-2 D_{r i}^{s}\right)\right. \\
\left.-K_{i}^{r}\left(D_{r s}^{s}-2 D_{s r}^{s}\right)\right]+2\left(B_{i}^{r}-\delta_{i}^{r} \operatorname{tr} B\right) V_{r} \\
+2\left(D_{r i}^{s}-\delta_{i}^{s} D_{j r}^{j}\right) B_{s}^{r} .
\end{gathered}
$$

Then one can relax the algebraic condition (19) and consider $V_{i}$ as a set of supplementary independent quantities to be evolved according to their evolution equations (24). The vector array $\mathbf{u}$ representing the independent quantities satisfying the balance law equations (18) will then contain the 37 functions

$$
\mathbf{u}=\left(\begin{array}{lllll}
\alpha, & \gamma_{i j}, \quad K_{i j}, \quad A_{i}, \quad D_{r i j}, & V_{i}
\end{array}\right)
$$

so that the condition (19) can now be considered as an algebraic constraint which will hold if and only if the momentum constraint is satisfied. This is the key point to get a hyperbolic system, and it has nothing to do with the coordinate gauge: it is just making a free use of the momentum constraint, a feature which is shared by other recent hyperbolic formulations $[20,21]$. The conditions under which this system is actually hyperbolic will be given below, along with the explicit eigenvectors and eigenvalues (characteristic speeds) and the diagonalized system.

\section{Invariant algebraic slicing}

Before we can complete our analysis, we need to know how the "slicing source function" $Q$, and how the shift vector $\beta^{i}$, depend on the fields to be evolved. We will use the lapse function degree of freedom to specify a time coordinate, which amounts to specifying $Q$. This will be done by relating the lapse to the space metric coefficients (dynamical lapse), but keeping the freedom of choosing arbitrary space coordinates on every slice (kinematical shift). We will demand then our lapse to be an algebraic condition, invariant under any transformation of the space coordinates on every slice. We must use then scalars, such as $\alpha, Q, \operatorname{tr} K$ and their proper time derivatives. If we restrict ourselves to scalars containing no derivatives of the metric coefficients, we can play only with $\alpha$ and we get either a "geodesic slicing" ( $\alpha=$ const) or one of its generalizations. This is too restrictive, as we will see later. If we allow also for first-order derivatives of the metric, we have also $Q$ and $\operatorname{tr} K$ at our disposal. As we have seen in the previous section, the principal part of the evolution system is quasilinear, so let us take a generic quasilinear homogeneous condition [1]

$$
Q=f(\alpha) \operatorname{tr} K,
$$

where $f$ is an arbitrary function.

The geodesic slicing is then included as a subcase with $f=0$. The $f=1$ case corresponds to the "harmonic slicing" $[22,26]$ (the resulting time coordinate is harmonic). Another interesting case is the " $1+\log$ " slicing [24,25], obtained when $f=1 / \alpha$; it mimics maximal slicing near a singularity, when the lapse collapses to zero. [Here we have considered the case $\alpha \propto 1+\ln (\sqrt{\gamma})$, which differs slightly from that considered in $[24,25]$. Both cases are included in this class of slicings.]

The slicing condition (26) can be integrated in normal coordinates (zero shift) to obtain, up to some integration constant,

$$
\sqrt{\gamma}=F(\alpha),
$$

where $F$ is an arbitrary function. This shows the generality of this condition, which is somehow hidden in its invariant form (26).

The widely used maximal slicing $[3,29](\operatorname{tr} K=0)$ is included also as a limiting case when $f$ diverges ( $F$ is constant). It is a very special case because the lapse $\alpha$ is given by an elliptic condition, so that the evolution system becomes a coupled hyperbolic-elliptic system. Moreover, $\alpha$ is no longer related to the space volume element $\sqrt{\gamma}$ (which is 
actually constant in the zero shift case) and the recent discussion of "coordinate shocks" [27] does not apply to maximal slicing.

It is worth studying the behavior of the slicings defined by Eq. (26) when the space volume element $\sqrt{\gamma}$ goes to zero. This will be a singularity of the slicing, but it is more convenient to view it in an equivalent way, as a singularity of the congruence of time lines normal to the slicing, so that we can use the integral form (27). Let us suppose that this singularity occurs after a finite proper time interval $\tau_{s}$ away from our initial time slice. The elapsed coordinate time will be given by the integral

$$
\Delta t=\int_{0}^{\tau_{s}} \frac{d \tau}{\alpha}
$$

so that an obvious necessary condition for singularity avoidance is that the lapse function vanishes before or at the singularity (lapse collapse), because otherwise the integral (28) will be finite and this means that the singularity will be reached in a finite coordinate time.

If the lapse vanishes (lapse collapse) at $\tau_{0}<\tau_{s}$, the slicing is said to have a "limit surface" and it stops before reaching the singularity: this happens for instance with maximal [30] or " $1+\log$ " slicing. If the lapse vanishes precisely at $\tau_{0}=\tau_{s}$, singularity avoidance would mean that the improper integral (28) diverges (one does not reach $\tau_{s}$ in a finite time). One can obtain a sufficient condition for singularity avoidance for "focusing singularities" [26], that is when the space volume element vanishes at a bounded rate, so that

$$
\left|\partial_{\tau} \sqrt{\gamma}\right|_{\tau_{s}}=\left|F^{\prime}(\alpha) \partial_{\tau} \alpha\right|_{\tau_{s}}<B
$$

and it is clear that if we assume strict monotonicity of $F$ at the singular point

$$
\left|F^{\prime}(\alpha)\right|_{\tau_{s}} \neq 0
$$

that would imply that the lapse itself vanishes at a bounded rate

$$
\left|\partial_{\tau} \alpha\right|_{\tau_{s}}<B^{\prime},
$$

and the improper integral (28) would not converge: the singularity cannot be reached in a finite coordinate time. It follows that focusing singularities are avoided by strictly monotonic choices of $F$, such as the ones that ensure hyperbolicity, as we will see below.

\section{E. Causal structure of the evolution system}

In what follows we will analyze the causal structure of the set of equations we have derived. It will turn out that under certain conditions, the system is hyperbolic, allowing a better understanding of the theoretical properties of the system that also permits yet more powerful numerical methods to be applied to the Einstein equations. In this section we consider the shift vector $\beta^{i}$ as a known function of spacetime. In the next section we discuss the shift and its effect on the system more fully.
The causal structure of a first-order system is given by its principal, or transport, part. The source terms contain no space derivatives, so that the principal part is given by the flux terms

$$
\partial_{t} \mathbf{u}+\partial_{k} F_{-}^{k} \mathbf{u}=0 .
$$

In this kind of analysis it is essential to write the entire system so that the source terms contain no derivatives of the fields. Otherwise, by manipulating the flux (derivative) and source terms one could apparently change the causal structure of the system at will. We can consider the transport part separately by splitting the evolution described by Eq. (18) into two separate processes: the first one is the transport process described by Eq. (32), and the second one is the sources contribution, given by the following system of ordinary differential equations

$$
\partial_{t} \mathbf{u}=S_{-} \mathbf{u} .
$$

This conceptual splitting can be easily implemented in numerical applications. If we note by $E(\Delta t)$ the numerical evolution operator for system (18) in a single time step, we get that, up to second-order accuracy in $\Delta t$,

$$
E(\Delta t)=S(\Delta t / 2) T(\Delta t) S(\Delta t / 2),
$$

where $T, S$ are the numerical evolution operators for systems (32) and (33), respectively. This is known as "Strang splitting" [31].

Note that, according to Eq. (17) the evolution equations for the lapse and the induced metric have no flux terms. This means that we can regard the transport step as the propagation of a reduced set of 30 quantities:

$$
\mathbf{u}=\left(K_{i j}, \quad A_{i}, \quad D_{r i j}, \quad V_{i}\right)
$$

in an inhomogeneous "background." The Eq. (32) is linear in the quantities (35), and this is a key point in what follows. This means that during the transport step the "background" quantities, which evolve according to Eq. (33), are fixed.

The standard procedure for studying the causal structure of first-order systems starts by choosing a fixed space direction. Only space derivatives along this direction will be considered, so that the resulting system is actually one dimensional. This procedure does not match the usual one for second order equations, where there is no need for choosing a priori a direction and all derivatives are dealt with simultaneously. The first order formalism, in contrast, allows one to treat one direction at a time, and this "locally onedimensional" (LOD) approch is useful both for theoretical analysis and numerical applications.

Let us begin our LOD analysis by taking for instance our space direction along the $x^{k}$ coordinate axis. We will then neglect all fluxes along the other directions $x^{k^{\prime}}$. It follows that, apart from the background metric coefficients, the 14 quantities

$$
A_{k^{\prime}}, D_{k^{\prime} i j}\left(i, j=1,2,3 k^{\prime} \neq k\right)
$$

have no flux along the $x^{k}$ direction, so that they are charac- 
teristic fields propagating along the time lines (zero characteristic speed). Propagation along time lines is much more convenient for numerical applications than propagation along normal lines $\left(\right.$ speed $-\beta^{k}$ ) as we had in our previous Letter [1]. [As we will see below, propagation along time lines can be treated by methods for ordinary differential equations (ODE's).] They only coincide in the zero shift case.

Rather than studying the transport properties of the u's, the evolution of the remaining 16 quantities is more easily studied by taking their fluxes (20) to be the basic quantities. Hence we rewrite the LOD transport equations using the fluxes along the selected direction $x^{k}$ as the basic quantities. We find that (the principal part of) the resulting equations can be written as a system of 16 one-dimensional advection equations (no sum in $k$ ):

$$
\partial_{t}\left(\begin{array}{l}
F_{-}^{k} V^{k} \\
F_{-}^{k} V_{k^{\prime}} \\
F_{-}^{k} K_{i k^{\prime}} \\
F_{-}^{k} D_{k i k^{\prime}} \\
F_{-}^{k} K_{r}^{r} \\
F_{-}^{k} A_{k} \\
F_{-}^{k} D_{k r}^{r}
\end{array}\right)+\mathbf{A} \partial_{k}\left(\begin{array}{l}
F_{-}^{k} V^{k} \\
F_{-}^{k} V_{k^{\prime}} \\
F_{-}^{k} K_{i k^{\prime}} \\
F_{-}^{k} D_{k i k^{\prime}} \\
F_{-}^{k} K_{r}^{r} \\
F_{-}^{k} A_{k} \\
F_{-}^{k} D_{k r}^{r}
\end{array}\right)=0,
$$

where $\mathbf{A}$ is the characteristic matrix of this reduced system

$$
\mathbf{A}=\left(\begin{array}{ccccccc}
-\beta^{k} & 0 & 0 & 0 & 0 & 0 & 0 \\
0 & -\beta^{k} & 0 & 0 & 0 & 0 & 0 \\
-n / 2 \alpha \gamma_{i k^{\prime}} & \alpha \delta_{i}^{k} & -\beta^{k} & \alpha \gamma^{k k} & 0 & 0 & 0 \\
0 & 0 & \alpha & -\beta^{k} & 0 & 0 & 0 \\
(2-3 n / 2) \alpha & 0 & 0 & 0 & -\beta^{k} & \alpha \gamma^{k k} & 0 \\
0 & 0 & 0 & 0 & \alpha f & -\beta^{k} & 0 \\
0 & 0 & 0 & 0 & \alpha & 0 & -\beta^{k}
\end{array}\right) .
$$

Its eigenvalues are then the "characteristic speeds." The corresponding right eigenvectors are the "characteristic fields." (38):

Let us list the 16 characteristic fields associated with Eq.

The three quantities $F_{-}^{k} V_{i}$ plus the single quantity $F_{-}^{k} A_{k}-f F_{-}^{k} D_{k r}^{r}$, which propagate along normal lines (speed $\left.-\beta^{k}\right)$.

The ten combinations

$$
F_{-}^{k} K_{i k^{\prime}} \pm \sqrt{\gamma^{k k}}\left[F_{-}^{k} D_{k i k^{\prime}}+\left(\delta_{i}^{k} F_{-}^{k} V_{k^{\prime}}-n / 2 \gamma_{i k^{\prime}} F_{-}^{k} V^{k}\right) / \gamma^{k k}\right] \text {, }
$$

which propagate along light cones (speed $-\beta^{k} \pm \sqrt{\gamma^{k k}}$, respectively).

The two combinations

$$
\sqrt{f} F_{-}^{k} K_{r}^{r} \pm \sqrt{\gamma^{k k}}\left[F_{-}^{k} A_{k}+(2-3 n / 2) F_{-}^{k} V^{k} / \gamma^{k k}\right]
$$

which propagate, respectively, with the gauge-dependent speed $-\beta^{k} \pm \sqrt{f \gamma^{k k}}$ ("gauge speed").

A system is said to be hyperbolic if all the characteristic speeds are real and the characteristic matrix can be fully diagonalized (see for instance Ref. [18]). This is our case provided that $f>0$ [note that if $f=0$, as in the geodesic case, the last combination (40) contains only one independent quantity and the set of eigenfields is no longer complete]. Gauge speed coincides with light speed only in the harmonic case $(f=1)$. It becomes infinite for a maximal slicing, which can be considered as a limiting case of our condition (26).

The advantage of having a hyperbolic system is that we know now explicitly which combination is propagating for- ward or backward along the selected direction. Suppose, for instance, that one is using a 3D Cartesian finite difference grid with vanishing shift at the outer boundaries. It follows that the five combinations one gets from Eq. (39) by using the plus (respectively minus) sign are entering the grid through the left (respectively right) outer boundary along the $x^{k}$ direction. The same thing happens with the gauge combination (40). This information should be very valuable when devising boundary conditions, as we will show in a future publication.

The very existence of "gauge speeds" is a remarkable result. One is used to thinking that light cones are enough to determine the causal structure of spacetime. This is true if we refer only to the invariant features. But the evolution system evolves spacetime together with the coordinate system we are using to label it (the dynamical lapse in our case). For instance, maximal slicing is associated with an infinite gauge speed (as it must be, because both the lapse and its derivatives are provided by an elliptic equation). These considerations single out the harmonic slicing, in which gauge cones and light cones do coincide. This further degeneracy simplifies the causal structure of spacetime, but it is just accidental and we see no reason to overlook the richer structure that arises in the general case.

\section{F. The role of the shift}

The balance law evolution equations for the entire set of variables (25) is valid for any choice of lapse function $\alpha$ and shift vector $\beta^{i}$ whatever. In the analysis of the causal structure, we considered very carefully the effect of the choice of lapse, and showed that for a large family of conditions, the 
system is actually hyperbolic. But so far we have said little about the shift, treating it as a given spacetime function. In such a case it has no dynamics and hence it does not enter in to the discussion of the causal structure of the system, except through its connection with the eigenspeeds of the other variables.

However, in practice in numerical relativity, the shift is not prescribed ahead of time as a known function of the spacetime coordinates. Instead, one wants it to respond to the dynamics of the system, and it is usually related in some explicit functional way to the metric and extrinsic curvature variables themselves. If the shift is taken to be some explicit function of the other fields, and introduced to the system in a way that it changes continuously as a dynamical variable, this could affect the causal structure of the system. In this case, one would need to substitute this prescription into the complete system and analyze the causal structure on a case by case basis. In all cases, the equations are still valid, but as far as the causal structure is concerned, for some cases the eigenfields and eigenspeeds could change, or the hyperbolicity itself could be broken. This statement should be true for any formulation of the Einstein equations. A careful analysis of many such cases, where the shift is considered as a true dynamical variable, has been carried out [32-34].

On the other hand, the shift need not be considered as a dynamical variable of the system. One is free to choose it any time as one likes. For example, on a given time slice one could prescribe it to be any arbitrary function of the other fields in the system, and hold this fixed as long as one likes. While the other variables in the system evolve, the shift is held fixed in time. Then the shift has no dynamics, and cannot affect the causal structure of the system. On a later time slice, one may again choose the shift freely, and then consider it to be fixed for the next period of evolution. In fact, one can do this as often as one likes, say on every time slice. However, it is crucial to point out that this is not the same as having a dynamic shift that can affect the causal structure of the system. In the latter dynamic case, the shift changes continuously as the other fields evolve. In the case we are considering, which we could call a momentarily "frozen shift," the evolution of the fields is decoupled from the development of the shift, and vice versa. During the evolution of the fields the shift has no dynamics, and is a known function of space. This is a subtle, yet crucial point. It is not merely a point of view, but a key practical point to be made. The shift can be chosen in this way on every time step, but in the process of evolving the fields from one time step to the next it must be regarded as a fixed function. Note that we can not say the same about the lapse, because this will amount to drop out the lapse derivatives $A_{k}$ [their fluxes in Eq. (37)] from the list of dynamical quantities. This means that we should then suppress the corresponding row and column in the characteristic matrix (38), that will no longer be diagonalizable.

As in this treatment the shift is a known function of space for all evolution steps, the previous analysis of the causal structure and hyperbolicity carries through for all shift choices one cares to make on all time steps. However, this treatment has certain numerical consequences that will be considered in the next section.

\section{NUMERICAL METHODS}

In this section we describe in broad terms the kind of numerical methods that can applied to this formulation of the Einstein equations. We will defer a detailed treatment, complete with numerical examples, to future publications. In the previous sections, we have reformulated the evolution equations as a first-order system of balance laws, valid for any choice of lapse and shift, without taking any additional derivative. This allows one to use standard numerical methods from computational fluid dynamics: Lax-Wendroff, staggered leapfrog, MacCormack [31]. We have also seen that for some choices of the lapse function the evolution system is hyperbolic, so that we can use more advanced numerical methods [18] and have better control at the boundaries, as we will see below.

To obtain a finite difference version of our equations, one can use the numerical splitting approach as we outlined above [see, e.g., Eq. (34)]. This has many advantages, which we summarize here:

The nonlinear terms in Einstein equations (the ones containing products of first derivatives of the metric) appear only in the sources step (33), in the form of a coupled system of ordinary differential equations. In numerical tests we have discretized this part using standard predictor-corrector or modified midpoint methods [31] to second-order accuracy, although many prescriptions are possible. Note that this gives one the possibility of using methods for stiff ODE's if the source terms behave in this way.

The remaining terms (the principal part) are in flux conservative form (32). This allows us to apply the highresolution methods which have been developed for computational fluid dynamics. We have discretized that part using a second order TVD (total variation diminishing) method [18], although again many possibilities could be examined.

We can tailor the shift to fit our needs by choosing any profile just after every sources step, but keeping it constant during the whole transport step ("frozen"' shift). This allows of course an explicit prescription of the shift (we have used for instance a parabolic shift profile to track the horizon of 1D black holes). But this also allows an indirect prescription, as it can be done for instance via the solution of some elliptic equation (minimal distortion shift, or similar conditions), exactly in the same way we do when imposing the maximal slicing condition for the lapse. The key point is that the shift must be kept fixed during the transport step, as described above, or else the analysis of the eigenfields would have to be redone.

Allowing for Eq. (17) and the previous considerations, the metric coefficients do not evolve in the transport step. This means that we are just evolving now the reduced set of dynamical variables (35) and the principal part (32) is linear in these quantities:

$$
F_{-}^{k} \mathbf{u}=\mathbf{A}^{k}(x) \mathbf{u}
$$

We can then describe the transport step by writing Eq. (32) in the form

$$
\partial_{t} \mathbf{u}+\partial_{k}\left[\mathbf{A}^{k}(x) \mathbf{u}\right]=0,
$$


because the metric coefficients contained in the matrix $\mathbf{A}^{k}$ are time independent in the transport step. The resulting equation (42) can be then interpreted as a transport equation in an inhomogeneous (but fixed) background. The alternative form (37) for the evolution of the fluxes (41) follows then easily.

The complete numerical details of $1 \mathrm{D}$ and 3D codes developed to solve these equations with a variety of advanced methods will be given elsewhere. It is our purpose to outline all details necessary to understand both theoretical and practical aspects of our approach, and to provide some simple examples of its power.

Before moving to more specific application in the 1D case, we further discuss an important numerical aspect of the treatment of the shift. As we have stressed, the equations we have presented are valid for any choice of lapse and shift, and a host of numerical methods may be applied as described. For applications where hyperbolicity is important, it is clear that the system is hyperbolic for a certain family of slicings and a given spacetime function of shift. Furthermore, any prescription for the shift, even if it is explicitly a function of the fields $\mathbf{u}$ and their derivatives, can preserve hyperbolicity if the system is treated appropriately by holding the shift fixed during the transport step.

The numerical implication of this treatment is important. It means that if hyperbolicity is desired, in some cases the shift may only be enforced numerically in a first-order way during the evolution. For example, in a method that requires a prediction and correction of the fields during the transport step, the shift must remain decoupled (fixed) during both steps. Hence although the fields may be evolved forward in time in a second-order way, in our present scheme the shift itself is not necessarily implemented to second-order accuracy in time. This issue only arises if one exploits information about the eigenfields in the actual numerical implementation. For example, in a MacCormack method, which does not make use of hyperbolicity, this is not an issue, nor is it an issue if the shift is a given spacetime function. The convergence properties for complicated examples, where the shift is prescribed as an explicit function of the fields (say, minimal distortion shift), and where knowledge of hyperbolicity is used in the numerical methods, will have to be investigated carefully in the future.

\section{A. The one-dimensional case}

Let us further illustrate the advantages of our formulation by outlining numerical methods in the one-dimensional case. The 3D case can be handled similarly by directional splitting. To label the values, we will use a superindex for the time level and a subindex for the grid position. For the transport equations

$$
\partial_{t} \mathbf{u}+\partial_{x} F_{-} \mathbf{u}=0,
$$

we can then use flux-conservative numerical methods of the form

$$
u_{i}^{n+1}=u_{i}^{n}-\frac{\Delta t}{\Delta x}\left(F_{i+1 / 2}^{n+1 / 2}-F_{i-1 / 2}^{n+1 / 2}\right)
$$

These are two-step algorithms. In the first step, one must compute the fluxes at the "interface points" $x_{i \pm 1 / 2}$ at the intermediate time level $t^{n+1 / 2}$ to a given accuracy (we will use first order). The final step (44) improves the overall accuracy by one order (we get then second-order overall accuracy). The time step is limited by the causality condition (also known as Courant-Friedrichs-Lewy condition [31]), which states that the maximal speed in the numerical algorithm is just $\Delta x / \Delta t$ (one grid point at a time), providing an upper bound to the time step $\Delta t$ to ensure that this speed is greater than the characteristic speeds.

In order to compute the interface fluxes $F_{i+1 / 2}^{n+1 / 2}$, we will take advantage of the fact that, allowing for Eq. (42), the transport equation (43) can be written as

$$
\partial_{t} \mathbf{F}+\mathbf{A}(x) \partial_{x} \mathbf{F}=0,
$$

which is the one-dimensional version of the system of advection equations (37). This means that the eigenfields of the characteristic matrix A (38) propagate along characteristic lines in either the forward or backward direction, depending on the sign of their characteristic speed. The fluxes can be first evaluated at every grid point, and then the diagonal combinations (i.e., the eigenfields) are computed. These diagonal combinations are propagated to the grid interfaces, using Eq. (45), and inverted there through the diagonalization process to compute the fluxes $F_{i+1 / 2}^{n+1 / 2}$ that we will need in the second step (44).

There are many ways to propagate the diagonal fluxes. Let us consider for instance a diagonal flux $F$ with positive characteristic speed $\lambda$. Let us decompose the resulting interface flux as

$$
F_{i+1 / 2}^{n+1 / 2}=F_{i}^{n}+\delta_{i},
$$

so that $F_{i}^{n}$ would be the zero-order prediction and $\delta$ gives then the difference between the zero and first or higher order estimates. Different $\delta$ values will lead to different methods, such as the Lax-Wendroff method,

$$
\delta_{i}^{\mathrm{LW}}=\frac{1}{2}\left(1-\lambda \frac{\Delta t}{\Delta x}\right)\left(F_{i+1}^{n}-F_{i}^{n}\right)
$$

or the Beam-Warning method,

$$
\delta_{i}^{\mathrm{BW}}=\delta_{i-1}^{\mathrm{LW}}=\frac{1}{2}\left(1-\lambda \frac{\Delta t}{\Delta x}\right)\left(F_{i}^{n}-F_{i-1}^{n}\right)
$$

and we see that, up to a factor, $\delta_{i}$ is a measure of the flux slope at the upstream grid point $x_{j}$.

One can choose a slope which is a nonlinear average of the two one-sided linear estimates; namely,

$$
\delta_{i}=\operatorname{minmod}\left(\delta_{i-1}^{\mathrm{LW}}, \quad \delta_{i}^{\mathrm{LW}}\right) .
$$

which corresponds to the "minmod" average (just discard the steepest slope). This leads to the "monotonic upstreamcentered" (MUSCL) algorithm, which is very robust and easy to implement. Unlike the Lax-Wendroff or BeamWarning methods, it has the TVD property [18], which means that no spurious numerical oscillations can appear during the transport step, and this proves to be very conve- 
nient in black hole codes, where large gradients arise near the horizon. We will show examples in a future publication.

\section{B. Boundary conditions}

The main problems with boundary conditions are consistency and stability. Hyperbolic systems show here one of their main advantages, because they allow a complete analysis of the information flow at the boundary. One can actually compute the characteristic fields at every boundary and separate them into two sets: either incoming or outgoing ones. Boundary conditions should provide external information for incoming fields only. Imposing any condition on outgoing fields would be inconsistent and boundary instability would arise in the code. In this sense, an "outgoing radiation" condition should be understood and implemented as a "no incoming radiation" condition for the incoming fields.

The second-order accurate operator splitting (34) that we are using requires boundary conditions to be imposed on the transport step only (the sources step is made of ordinary differential equations). This is why we have chosen the ordering given in Eq. (34) instead of

$$
E(\Delta t)=T(\Delta t / 2) S(\Delta t) T(\Delta t / 2),
$$

which would require to impose boundary conditions twice on a single time step.

The natural way to impose boundary conditions in the two step schemes (44) that we are using for the transport part is by prescribing the fluxes $F^{n+1 / 2}$ of the incoming fields at the outermost interfaces

$$
F_{i \min -1 / 2}^{n+1 / 2}, \quad F_{i \max +1 / 2}^{n+1 / 2},
$$

where $i$ min, $i$ max are the labels of the boundary points.

We have seen in the previous sections that, for every space direction, there are six incoming eigenfields at the outer boundaries (five on the light cone plus one on the gauge cone). Remember, however, that we have three "redundant" quantities $V_{i}$ in our formalism, which are related to the metric derivatives through the algebraic equation (19), which amounts to the momentum constraint. This allows us to use the computed values of the $V_{i}$ to reduce the number of algebraically independent incoming fields at the boundary to what one could expect from physical considerations: one gauge field plus two light cone fields which account for the gravitational radiation degrees of freedom.

One could argue that the use of the constraint (19) at the boundaries is not consistent in the operator splitting approach, because the constraints are not first integrals of the transport and source parts, when considered separately. This is true in the generic case, but one should notice that in the Strang splitting we are using, Eq. (34), we impose the boundary conditions at $t^{n+1 / 2}$ in the transport step, so that we have evolved, precisely,

$$
T(\Delta t / 2) S(\Delta t / 2)
$$

and the values $u^{n+1 / 2}$ provide a consistent first order approximation of the complete equation (source terms included) at $t^{n+1 / 2}$.

\section{CONCLUSIONS}

We have extended the discussion of our previous Letter on a new hyperbolic formulation of Einstein's equations for numerical relativity. The equations have been cast into a flux conservative system of balance laws that is valid for any choice of lapse and shift. This formulation allows one to use numerical methods such as those of MacCormack, LaxWendroff, and others. We further showed that the system of equations is hyperbolic, meaning that its characteristic matrix is fully diagonalizable with real eigenvalues, for a wide variety of lapse conditions, including most of those commonly used in numerical relativity. This hyperbolicity can be maintained for arbitrary shift choices, provided that care is taken in the numerical implementation during the transport step of the evolution scheme.

This hyperbolicity of the system can provide many additional benefits, including the application of a large body of numerical methods that make use of the eigenfields and their characteristic speeds. Such methods have been crucial in hydrodynamics, where large gradients develop, and should find application in numerical relativity as well. We sketched in a general way how some of these methods can be applied to our system of equations. Hyperbolicity can also provide information about the fields, their direction of propagation, and their speeds, that should prove to be very useful in devising boundary conditions, both at horizons (AHBC) and at numerical grid boundaries. In both cases, one wants to enforce that outgoing signals leave the boundary, and that no spurious signals come in.

Finally, we showed that our system of equations can be modified by use of the Hamiltonian constraint to remove numerically difficult terms in the evolution equations, while maintaining hyperbolicity. This leads us to propose use of what we refer to as the "Einstein" system, as opposed to the standard "Ricci" system. Detailed numerical examples of these ideas will be provided in future papers in this series.

\section{ACKNOWLEDGMENTS}

We have benefited from discussions with a number of our colleagues, particularly Wai-Mo Suen. This work was supported by the Dirección General para la Investigación Científica y Técnica of Spain under project PB94-1177, and also by NSF Grant Nos. PHY94-07882 and INT94-14185. 
[1] C. Bona, J. Massó, E. Seidel, and J. Stela, Phys. Rev. Lett. 75, 600 (1995).

[2] C. Bona and J. Massó, Phys. Rev. Lett. 68, 1097 (1992).

[3] A. Lichnerowicz, J. Math. Pures Appl. 23, 37 (1944).

[4] Y. Choquet-Bruhat, in Gravitation: An Introduction to Current Research, edited by L. Witten (Wiley, New York, 1962).

[5] R. Arnowitt, S. Deser and C. W. Misner, in Gravitation: An Introduction to Current Research [4].

[6] E. Seidel and W.-M. Suen, Phys. Rev. Lett. 69, 1845 (1992).

[7] P. Anninos, G. Daues, J. Massó, E. Seidel, and W.-M. Suen, Phys. Rev. D 51, 5562 (1995).

[8] D. Bernstein, D. Hobill, and L. Smarr, in Frontiers in Numerical Relativity, edited by C. Evans, L. Finn, and D. Hobill (Cambridge University Press, Cambridge, England, 1989).

[9] S. L. Shapiro and S. A. Teukolsky, in Dynamical Spacetimes and Numerical Relativity, edited by J. M. Centrella (Cambridge University Press, Cambridge, England, 1986).

[10] S. L. Shapiro and S. A. Teukolsky, Phys. Rev. Lett. 66, 994 (1991).

[11] C. Bona, J. M. Ibáñez, J. M. Martí, and J. Massó, in Gravitation and General Relativity: Rotating Bodies and Other Topics, edited by F. Chinea and L. M. Gonzalez-Romero (Lecture Notes in Physics, Vol. 423) (Springer Verlag, Berlin, 1993).

[12] R. W. MacCormack, in Proceedings of the Second International Conference on Numerical Methods in Fluid Dynamics, edited by M. Holt (Lecture Notes in Physics, Vol. 8) (Springer-Verlag, Berlin, 1971).

[13] A. Harten, SIAM (Soc. Ind. Appl. Math.) J. Numer. Anal. 21, 1 (1984).

[14] P. D. Lax and B. Wendroff, Commun. Pure Appl. Math. 13, 217 (1960).

[15] R. D. Ritchmeyer and K. W. Morton, Difference Methods for Initial-value Problems, 2nd ed. (Interscience, New York, 1967).
[16] S. K. Godunov et al., Resolution Numerique des Problemes Multidimensionnels de la Dynamique des Gaz (MIR, Moscow, 1978).

[17] G. A. Sod, Numerical Methods in Fluid Dynamics (Cambridge University Press, Cambridge, England, 1985).

[18] R. J. LeVeque, Numerical Methods for Conservation Laws (Birkhauser, Basel, 1992).

[19] Y. Foures-Bruhat, Acta Math. 88, 141 (1955).

[20] Y. Choquet-Bruhat and J. W. York, C. R. Acad. Sci. I, Math. 321, 1089 (1995); A. Abrahams, A. Anderson, Y. ChoquetBruhat and J. W. York, Phys. Rev. Lett. 75, 3377 (1995).

[21] S. Frittelli and O. A. Reula, Phys. Rev. Lett. 76, 4667 (1996).

[22] Y. Choquet-Bruhat and T. Ruggeri, Commun. Math. Phys. 89, 269 (1983).

[23] H. Friedrich, Commun. Math. Phys. 100, 525 (1985); Class. Quantum Grav. 13, 1451 (1996).

[24] A. Abrahams, D. Bernstein, D. Hobill, E. Seidel, and L. Smarr, Phys. Rev. D 45, 3544 (1992).

[25] P. Anninos, D. Hobill, E. Seidel, L. Smarr, and W.-M. Suen, Phys. Rev. Lett. 71, 2851 (1993).

[26] C. Bona and J. Massó, Phys. Rev. D 38, 2419 (1988).

[27] M. Alcubierre, Phys. Rev. D 55, 5981 (1997).

[28] P. Laguna (private communication).

[29] J. W. York, Jr., Phys. Rev. Lett. 28, 1082 (1972).

[30] F. Estabrook, H. Wahlquist, S. Christensen, B. DeWitt, L. Smarr, and E. Tsiang, Phys. Rev. D 7, 2814 (1973).

[31] W. H. Press, B. P. Flannery, S. A. Teukolsky, and W. T. Vetterling, Numerical Recipes, 2nd ed. (Cambridge University Press, Cambridge, 1992).

[32] C. Bona, J. Massó, J. Stela, and E. Seidel, in General Relativity (MG7 Proceedings), edited by R. Ruffini and M. Keiser (World Scientific, Singapore, 1995).

[33] W.-M. Suen (private communication).

[34] P. Anninos, G. Daues, J. Massó, E. Seidel, and W.-M. Suen, Phys. Rev. D 51, 5562 (1995). 